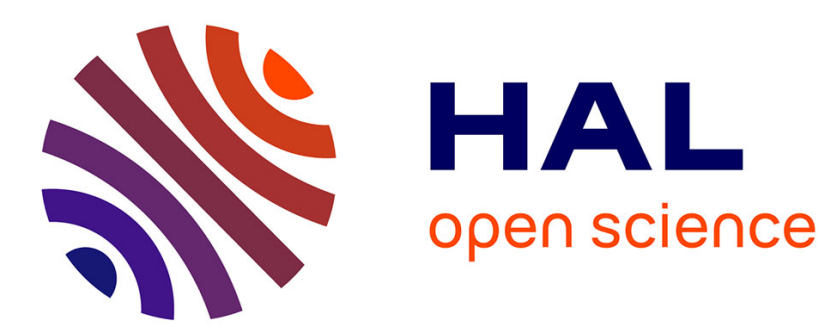

\title{
Ratiometric Nanoparticle Probe Based on FRET-Amplified Phosphorescence for Oxygen Sensing with Minimal Phototoxicity
}

Pichandi Ashokkumar, Nagappanpillai Adarsh, Andrey S Klymchenko

\section{To cite this version:}

Pichandi Ashokkumar, Nagappanpillai Adarsh, Andrey S Klymchenko. Ratiometric Nanoparticle Probe Based on FRET-Amplified Phosphorescence for Oxygen Sensing with Minimal Phototoxicity. Small, 2020, 16 (32), pp.2002494. 10.1002/smll.202002494 . hal-03085816

\section{HAL Id: hal-03085816 https://hal.science/hal-03085816}

Submitted on 22 Dec 2020

HAL is a multi-disciplinary open access archive for the deposit and dissemination of scientific research documents, whether they are published or not. The documents may come from teaching and research institutions in France or abroad, or from public or private research centers.
L'archive ouverte pluridisciplinaire HAL, est destinée au dépôt et à la diffusion de documents scientifiques de niveau recherche, publiés ou non, émanant des établissements d'enseignement et de recherche français ou étrangers, des laboratoires publics ou privés. 


\section{Article type: Full Paper}

\section{Ratiometric Nanoparticle Probe Based on FRET-Amplified Phosphorescence for Oxygen Sensing with Minimal Phototoxicity}

Pichandi Ashokkumar ${ }^{\xi \dagger}$, Nagappanpillai Adarsh ${ }^{\S}$ and Andrey S. Klymchenko*

Dr. P. Ashokkumar, Dr. N. Adarsh, Dr. A. S. Klymchenko

Laboratoire de Bioimagerie et Pathologies, UMR 7021 CNRS, Faculté de Pharmacie, Université de Strasbourg, Strasbourg CS 60024, France

${ }^{\S}$ P. A. and N. A. contributed equally to this work.

*Corresponding author: andrey.klymchenko@unistra.fr

†Present Address: Department of Bioelectronics and Biosensors, Alagappa University, Karaikudi 630003, India

Keywords: oxygen sensors; dye-loaded polymeric nanoparticles; phosphorescence; singleparticle fluorescence; oxygen gradients in microfluidics.

Abstract: Luminescent oxygen probes enable direct imaging of hypoxic conditions in cells
and tissues, which are associated with variety of diseases, including cancer. Here, we 
developed nanoparticle probe that addresses key challenges in the filed: it (i) strongly amplifies room temperature phosphorescence of encapsulated oxygen-sensitive dyes; (ii) provides ratiometric response to oxygen; and (iii) solves the fundamental problem of phototoxicity of phosphorescent sensors. The nanoprobe is based on 40-nm polymeric nanoparticles, encapsulating $\sim 2000$ blue-emitting cyanine dyes with fluorinated tetraphenylborate counterions, which are as bright as 70 quantum dots (QD525). It functions as a light-harvesting nanoantenna that undergoes efficient FRET to 20 phosphorescent oxygen-sensitive platinum octaethylporphyrin (PtOEP) acceptor dyes. The obtained nanoprobe emits stable blue fluorescence and oxygen-sensitive red phosphorescence, thus providing ratiometric response to dissolved oxygen. The light-harvesting leads to $\sim 60$-fold phosphorescence amplification and makes the single nanoprobe particle as bright as $\sim 1200$ PtOEP dyes. This high brightness enables detection of dissolved oxygen at a single-particle level and imaging oxygen in cells at ultra-low nanoprobe concentration with no sign of phototoxicity, in contrast to PtOEP dye. The developed nanoprobe is successfully applied to imaging of microfluidics-generated oxygen gradient in cancer cells. It constitutes a promising tool for bioimaging of hypoxia.

\section{Introduction}

Molecular oxygen has a crucial role to supply metabolic energy to cells in aerobic respiration. ${ }^{[1]}$ The deficiency of molecular oxygen, commonly referred as hypoxia, is directly related to cancer growth, neurological disorders, retinal diseases, etc, and the elevated levels of oxygen (hyperoxia) can lead to generation of reactive oxygen species and free-radicals. ${ }^{[2]}$ Among existing oxygen sensing methods, such as chemical ${ }^{[3]}$ and electrochemical, ${ }^{[4]}$ optical methods based on luminescent probes have attracted a growing attention in recent years, since they offer reversible responses with no electrical interferences and in a relatively noninvasive 
manner ${ }^{[5]}$ and allow fabricating devices based on films and optical fibers. ${ }^{[6]}$ In this case, phosphorescence is a key mechanism to achieve oxygen sensitivity, because the triplet excited state of dyes is efficiently quenched by oxygen. ${ }^{[7]}$ Particularly popular are palladium(II)- and platinum(II)-porphyrins, as well as complexes of iridium(III) and ruthenium(II). ${ }^{[5 a, 8]}$ However, their use as oxygen sensors meet a number of challenges, in particular quantification of the signal and phototoxicity. Although a number of phosphorescence-based oxygen probes have been developed, ${ }^{[5,9]}$ their intensity-based signal is difficult to quantify, so that sophisticated fluorescence lifetime detection scheme is needed. Ratiometric optical oxygen sensors are an attractive alternative, because intensity ratio, similarly to lifetime, provides robust absolute measure of the analyte and requires rather simple detection setup. ${ }^{[5 b, 10]}$ For this purpose, phosphorescent and fluorescent dyes are combined together to generate Förster Resonance Energy Transfer (FRET), where phosphorescent dyes (FRET acceptors) emit in the oxygendependent manner, and fluorescent dyes (donors) provide stable oxygen-independent reference signal. ${ }^{[5 b, 10]}$ Molecular, FRET-based probes were reported, ${ }^{[11]}$ however their design is challenging because of complex multistep synthesis, poor solubility in water and poor luminescent brightness due to donor-acceptor quenching. An attractive approach is to combine these phosphorescent dyes with fluorescent nanomaterials. The latter include conjugated polymer nanoparticles (NPs), ${ }^{[12]}$ quantum dots (QDs), ${ }^{[13]}$ carbon dots, ${ }^{[14]}$ dyedoped silica NPs, ${ }^{[15]}$ aggregation-induced emission $\mathrm{NPs}^{[16]}$ and dye-loaded polymeric NPs. ${ }^{[17]}$ So far, a variety of oxygen-sensitive probes combining fluorescent NPs and phosphorescent acceptor dye, has been reported mainly based on conjugated polymer NPs, ${ }^{[8 c, 18]}$ and, less commonly, dye-doped polymeric NPs ${ }^{[19]}$, core-shell polymeric $\mathrm{NPs}^{[20]}$ and supramolecular NPs. ${ }^{[21]}$ However, in these systems oxygen sensing by phosphorescent dyes generates highly reactive singlet oxygen, ${ }^{[22]}$ which makes this technique phototoxic. Therefore, many of these NPs were also proposed for photodynamic therapy applications. ${ }^{[8 c, 18 \mathrm{~d}]}$ To the best of our 
knowledge, design of bright nanoprobes for oxygen with minimized phototoxic effects of phosphorescent dyes remains an unexplored field.

New possibilities offer ultrabright fluorescent dye-loaded polymeric NPs, ${ }^{[17 a]}$ which already found different applications in single-particle tracking, ${ }^{[23]}$ super-resolution imaging, ${ }^{[17 b]}$ single-molecule detection, ${ }^{[24]}$ nucleic acid detection, ${ }^{[25]}$ as well as multicolor cellular and in vivo imaging. ${ }^{[26]}$ In these NPs, the problem of aggregation-caused quenching (ACQ) of encapsulated dyes was resolved by us using bulky fluorinated counterions that operate as fluorophore insulators. ${ }^{[17 \mathrm{~b}, 27]}$ The obtained dye-loaded polymeric NPs were up to 100 -fold brighter than QDs of similar color and size. ${ }^{[25 a, 28]}$ The other key feature is their exceptional FRET efficiency, where NPs loaded with $~ 10000$ dyes, so-called light-harvesting nanoantennas, can transfer energy to a single acceptor, thus amplifying its emission $>1000$ fold. ${ }^{[24]}$ Functionalization of these nanoantenna particles with DNA yielded nanoprobes for amplified detection of nucleic acids, which were $>2000$-fold brighter than reported FRETbased molecular probe, ${ }^{[25 a]}$ and sensitivity down to single molecules. ${ }^{[25 b]}$ Therefore, we hypothesized that the combination of these dye-loaded NPs with an oxygen-sensitive phosphorescent dye in form of a donor-acceptor FRET system would provide a facile route to oxygen sensors with drastically improved brightness. Moreover, owing to their high FRET donor capability, our NPs would require minimal number of phosphorescent dyes (acceptors) to obtain efficient FRET, which could decrease eventual phototoxic effects.

Herein, based on light-harvesting dye-loaded fluorescent nanoparticles that amplify phosphorescence of encapsulated oxygen-sensitive dye, we developed an oxygen sensor featuring high brightness, ratiometric response and low phototoxicity. We prepared $40 \mathrm{~nm}$ PMMA-MA NPs encapsulating an energy donor based on blue cyanine dye with bulky hydrophobic counterion (BlueCy-TPB) and oxygen-sensitive phosphorescent acceptor platinum octaethylporphyrin (PtOEP) (Figure 1). Efficient FRET from ca. 2000 donors to $c a$. 20 acceptors inside the particles was achieved, leading to 60 -fold phosphorescence 
amplification. The obtained nanoprobe emitted stable fluorescence as well as oxygensensitive red shifted phosphorescence, equivalent in brightness to 1200 PtOEP molecules, providing ratiometric response to dissolved oxygen. This high brightness enabled oxygen sensing at the single-particle level. The oxygen nanoprobe internalized inside cells showing no sign of phototoxicity, in contrast to PtOEP dye alone. Finally, in vitro mapping of dissolved oxygen gradients in HeLa cell culture was demonstrated by a specially designed microfluidic chamber.

A)

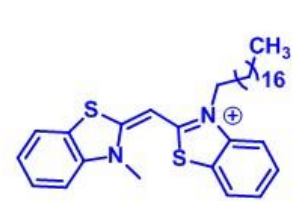

BlueCy

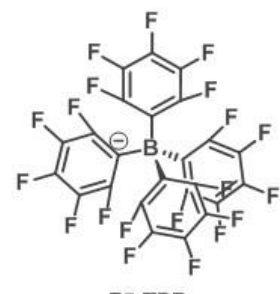

F5-TPB

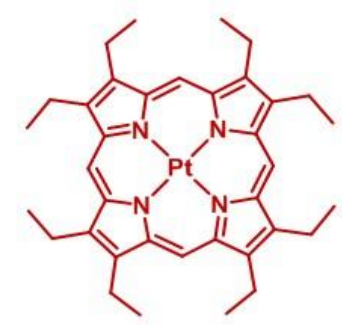

PtOEP

B)

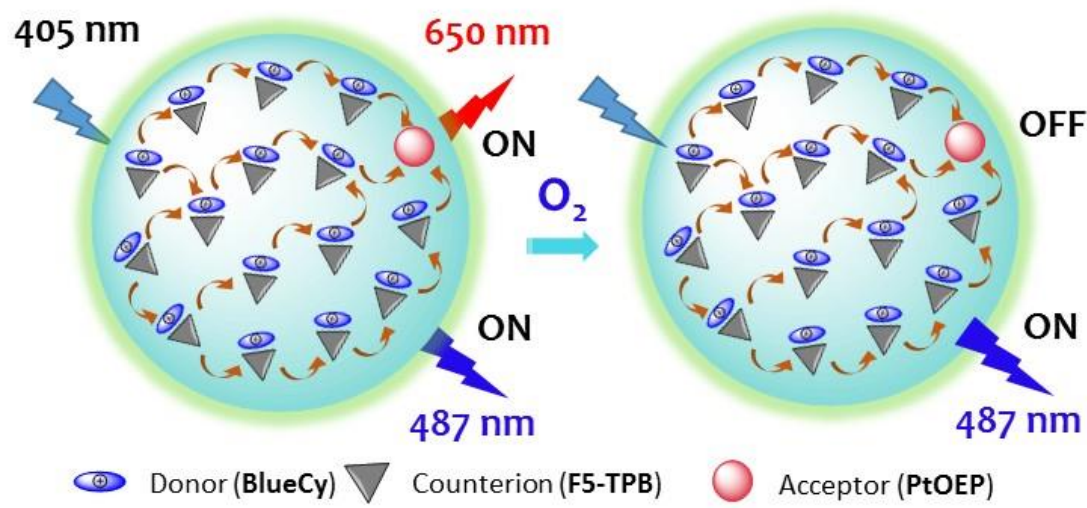

Figure 1. Concept of light-harvesting nanoparticle encapsulating BlueCy-TPB/PtOEP for ratiometric detection of oxygen. A) Molecular structures of the cyanine donor dye (BlueCy) with its counterion F5-TPB and of the porphyrin acceptor dye (PtOEP). B) Schematic representation FRET-based ratiometric oxygen nanoprobe. 


\section{Results and Discussion}

\subsection{Design of the oxygen nanoprobe}

As a polymer matrix for preparation of NPs, we selected PMMA-MA and PLGA, which were found very efficient for encapsulating different cationic organic dyes. ${ }^{[17 b, 17 c, 26,28]}$ PtOEP was chosen as a phosphorescent oxygen sensitive unit, which has already been used in oxygen sensors. ${ }^{[5 b, 18 b, 29]}$ However, in our nanoprobe, we plan to encapsulate PtOEP as FRET acceptor at very low concentration in the polymer matrix together with highly concentrated FRET donors, which will serve as a light-harvesting system for amplifying phosphorescence of PtOEP and generating ratiometric response to oxygen (Figure 1). Therefore, in this study the FRET donor should be carefully selected based on the following criteria: (1) ensure efficient FRET which requires good spectral overlap of the donor emission and the PtOEP absorption, centered around $530 \mathrm{~nm}$; (2) its emission should be well separated from that of PtOEP to provide a ratiometric output without any crosstalk and (3), the dye should be cationic, so that we could apply the counterion approach to prevent $\mathrm{ACQ}^{[17 \mathrm{~b}, 26-27]}$ and ensure efficient lightharvesting process. ${ }^{[24-25]}$ Therefore, we have chosen thiacyanine dye family, which operates in the blue spectral region. ${ }^{[30]}$ To provide proper encapsulation, a hydrophobic chain of 18 carbons was incorporated to the fluorophore (BlueCy). Moreover, the obtained dye BlueCy was paired with bulky hydrophobic couterion tetrakis(pentafluorophenyl)borate (F5-TPB), which has shown to be efficient against ACQ in many cationic dyes and to favor dye encapsulation into polymeric NPs. ${ }^{[17 b, 17 e, 26-27]}$

\subsection{Donor NPs}

The BlueCy dye was synthesized in three steps by modifying the reported procedure (Scheme S1). ${ }^{[30 a]}$ BlueCy derivative was obtained in a good yield by the condensation of quaternized 2- 
methyl-3-octadecylbenzo[d]thiazol-3-ium salt with quaternized 3-methyl-2(methylthio)benzo[d]thiazol-3-ium salt in the presence of trimethylamine in absolute ethanol. The counterion, iodide, was then exchanged with hydrophobic fluorinated anion, tetrakis(pentafluorophenyl) borate (F5-TPB) to obtain a target dye salt BlueCy-TPB. The final product and intermediates have been characterized by standard analytical procedures (Figure S1-S5).

BlueCy-TPB showed absorption and emission bands centered around 425 and $475 \mathrm{~nm}$, respectively, which is characteristic for the blue cyanine dyes with thiazole moities. ${ }^{[30 \mathrm{c}]}$ The dye exhibited negligible fluorescence with a quantum yield (QY) of $0.1-0.3 \%$ in organic solvents such as dioxane, methanol, etc (Table S1). Interestingly, a considerable enhancement in the fluorescence was observed in aqueous medium with a quantum yield of $6.1 \%$, probably due to the aggregation-induced emission (AIE). ${ }^{[16 a, 16 c-f, 31]}$ The aggregation in water was evidenced by the formation of a new absorption band at the shorter wavelength region (Figure 2A). To prove that the observed enhancement in the fluorescence intensity in aqueous medium arise from the restriction of molecular rotation in aggregates, ${ }^{[16 a, 31 b]}$ we measured fluorescence properties in highly viscous medium, glycerol. As expected, the dye showed marked enhancement in the quantum yield up to $21 \%$ (Table S1), which confirms the restriction of rotation around the methine bond between two benzothiazoles. The fluorescence enhancement is known for complexes of cyanine dyes with $\mathrm{DNA}^{\left[{ }^{[32]}\right.}$ as well as for other cationic dyes associated with bulky counterions. ${ }^{[27 c, 33]}$ In our case, this property is important for enhancing emission of the dye once it is encapsulated into the rigid polymer matrix of NPs. 

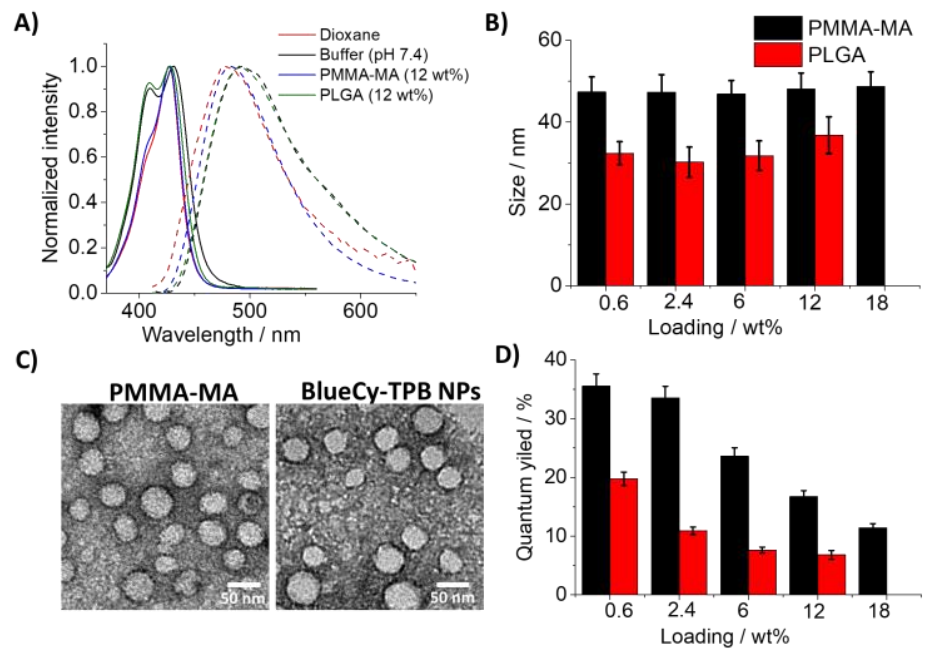

Figure 2. Characterization of BlueCy-TPB dye and its polymeric NPs. A) Absorption and emission spectra of BlueCy-TPB dye in dioxane, phosphate buffer ( $\mathrm{pH} \mathrm{7.4)} \mathrm{and} \mathrm{in} \mathrm{NPs} \mathrm{of}$ different loading. B) Plot showing the size by DLS for different loading of BlueCy-TPB. C) Representative TEM images of the PMMA-MA NPs with and without BlueCy-TPB (12 wt\% dye loading, counter stained with uranyl acetate). D) Dependence of fluorescence quantum yield on the dye loading.

Next, we prepared BlueCy dye-loaded polymeric NPs by using our recently developed protocol of charge-controlled nanoprecipitation. ${ }^{[17 a-c, 23]}$ For this purpose, a solution of the polymer (PLGA or PMMA-MA) and BlueCy-TPB in dioxane/acetonitrile mixture was rapidly added into aqueous media (20 mM phosphate buffer, $\mathrm{pH} 7.4$ ). The BlueCy-TPB is expected to co-precipitate together with the polymers due to its high hydrophobicity brought by the long C18 alkyl chain and the bulky hydrophobic counterion, leading encapsulation inside the particles. ${ }^{[17 \mathrm{a}-\mathrm{c}, 26-27,28]}$ Absorption spectra revealed that nanoprecipitation of the dye with PLGA or PMMA-MA resulted in the narrow absorption spectra, similar to that in dioxane, whereas in control conditions without the polymer, a clear blue-shifted shoulder appeared probably due to aggregation of the dye in the buffer (Figure 2A). These results provide the first indication that the dye was blended with the polymer after the nanoprecipitation. In case of PLGA and PMMA-MA, bearing a negatively charged carboxylate, NPs of $33 \pm 2$ and $45 \pm 2 \mathrm{~nm}$ diameter were formed, respectively, according to 
dynamic light scattering (DLS) (Figure 2B). The polymeric NPs maintained their monodispersity and small size upon increasing the BlueCy-TPB dye loading from 0.6 to 12 wt\% with respect to the polymer (Figure 2B, Table S2). In case of PMMA-MA NPs with 12 wt\% of BlueCy-TPB, the transmission electron microscopy (TEM) suggested the formation of uniform spherical particles with an average size of $40 \mathrm{~nm}$ (Figures $2 \mathrm{C}$ and S6), in line with the DLS data. Based on the diameter of NPs $(40 \mathrm{~nm})$ we can calculate particle volume (33500 $\mathrm{nm}^{3}$ ) and, then, assuming the BlueCy-TPB dye loading at $12 \mathrm{wt} \%$ (100 $\mathrm{mM}$ dye concentration) and polymer density of $1 \mathrm{~g} / \mathrm{ml}$, we can estimate that $\sim 2000$ donor dyes are encapsulated per NP. By contrast, DLS data revealed that BlueCy with small inorganic counterions such as iodide (BlueCy-I) showed drastic increase in the particle size with dye loading (Table S2). These data are in agreement with our earlier reports, showing that with small inorganic anions, the cationic dyes are adsorbed a the NPs surface leading to particle aggregation. ${ }^{[17 b, 27]}$ The QY values of the NPs were found to be as high as $35 \%$ for PMMAMA NPs at $0.6 \%$ dye loading. However, the increase in the dye loading produced a gradual decrease in QY of NPs, which can be due to ACQ at high dye loading (Figure 2D). In line with this observation, the mean lifetime of the BlueCy-TPB in PMMA-MA NPs also gradually decreased from $2.31 \pm 0.15 \mathrm{~ns}$ to $1.10 \pm 0.09 \mathrm{~ns}$ with the increase in the dye loading from 0.6 to 12 wt\% (Figure S7). At low BlueCy-TPB loading, the fluorescence decay was biexponential, dominated by the long-lived component (Table S3). At higher dye loading, new short-lived decay component appeared (0.16-0.18 ns) and the amplitudes of the short- and medium-lived components gradually increased. In addition, the decay time of long-lived component decreased from $2.53 \mathrm{~ns}$ to $1.63 \mathrm{~ns}$ with increase in dye loading from 0.6 to $12 \mathrm{wt} \%$. The observed multi-exponential decay at high dye loading, in line with earlier works on rhodamine-loaded NPs, ${ }^{[17 b, 24]}$ indicates a complex quenching process, which probably includes both dark states (corresponding to the short-lived component) and those quenched by excitation energy transfer (medium-lived component, $0.84 \mathrm{~ns}$ at $12 \mathrm{wt} \%$ loading). 
Nevertheless, the QY values remained relatively high in PMMA-MA NPs, namely 17\% even at 12 wt\% dye loading BlueCy-TPB, whereas the salt with small anion, BlueCy-I, in PMMA-MA NPs showed systematically lower QY values (Table S2). This result highlights the importance of the bulky hydrophobic counterion F5-TPB to prevent ACQ. ${ }^{[17 a, 17 b, 27 a, 27 b]}$ Moreover, BlueCy-TPB-loaded PMMA-MA NPs displayed significantly higher QY values compared to PLGA NPs (Figure 2D), which according to our earlier studies indicates lower level of dye clustering in PMMA-MA NPs. ${ }^{[28]}$ Therefore, in the next steps we focused our research on BlueCy-TPB-loaded PMMA-MA NPs.

Then, the single-particle fluorescence of the PMMA-MA NPs immobilized on the glass surface was characterized, using wide-field microscopy. BlueCy-TPB NPs appeared as fluorescent spots and their intensity increased with the dye loading (Figure 3). Remarkably, single-particle brightness of PMMA-MA with $12 \mathrm{wt} \%$ BlueCy-TPB was found to be 70-fold higher than that of QDs of similar emission wavelength (QD525). This result was supported by estimated theoretical brightness, ${ }^{[17 a]} \mathrm{N}$ (number of dyes) $\mathrm{x} \varepsilon$ (molar absorptivity) $\mathrm{x}$ QY (quantum yield): $2000(\mathrm{~N}) \times 6.7 \times 10^{4} \mathrm{M}^{-1} \mathrm{~cm}^{-1}(\varepsilon) \times 0.173(\mathrm{QY})=2.30 \times 10^{7} \mathrm{M}^{-1} \mathrm{~cm}^{-1}$ for BlueCy-TPB NPs (Table S4) and only $1(\mathrm{~N}) \times 3.70 \times 10^{5} \mathrm{M}^{-1} \mathrm{~cm}^{-1}(\varepsilon) \times 0.69(\mathrm{QY})=2.55 \times 10^{5}$ $\mathrm{M}^{-1} \mathrm{~cm}^{-1}$ for QD525 at $395 \mathrm{~nm}$ excitation (based on data from the provider, ThermoFisher Scientific). However, our NPs are 2-fold larger than QD525 ( 40 nm vs 15-20 nm), which should be taken into account in this comparison. On the other hand, the size of fluorescent core in our NPs can be tuned to much larger extent within $9-100 \mathrm{~nm},{ }^{[17 c, 23]}$ which makes them flexible for different applications. 
A)

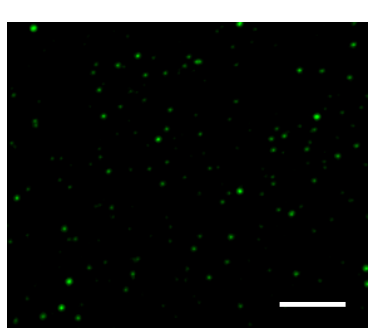

B)

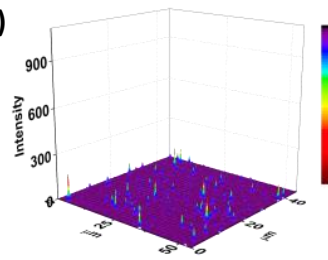

$2.4 \%$
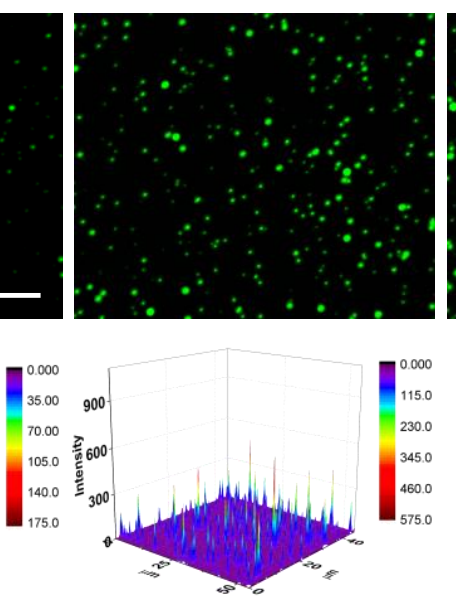

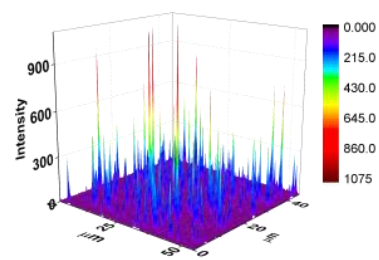

QD $525(x 10)$
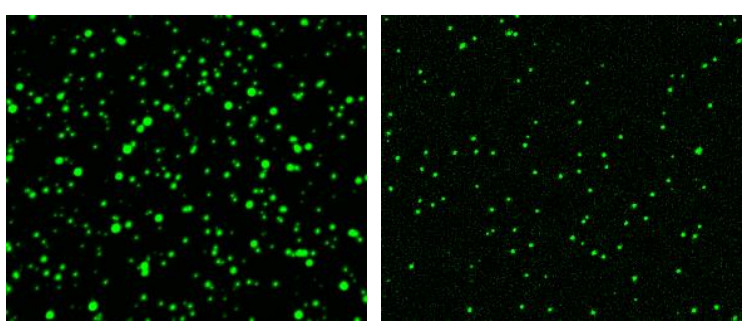

Figure 3. Single-particle evaluation of BlueCy-TPB NPs under wide-field fluorescence microscopy. A) Fluorescence images of NPs with different dye loading and QD525 immobilized on a glass surface. In the case of QDs, the intensity was multiplied by 10 -fold to make the signal visible. B) Corresponding 3D representation graphs. All imaging conditions were identical for dye-loaded NPs and QDs. The excitation wavelength is $395 \mathrm{~nm}$. Scale bar is $10 \mu \mathrm{M}$.

\subsection{Preparation and validation of oxygen nanoprobe}

Further, we prepared FRET NPs by introducing platinum octaethylporphyrin (PtOEP), an oxygen sensitive unit (Figure 1), acting as an energy acceptor for BlueCy-TPB. DLS and TEM data confirmed formation of small NPs around $40 \mathrm{~nm}$ size (Figure 4A, Figure S6). In the obtained NPs, the absorption spectrum of the PtOEP with a maximum at $~ 535 \mathrm{~nm}$ (Qband) overlapped efficiently with the emission profile of the donor, BlueCy-TPB (Figure S8), suggesting that this is a good FRET pair. Indeed, increase in the PtOEP loading in BlueCyTPB NPs (increase in the acceptor/donor ratio) decreased gradually the donor emission at 485 $\mathrm{nm}$, accompanied by an increase in the PtOEP emission band at $650 \mathrm{~nm}$, which is a clear indication of FRET from of BlueCy-TPB to PtOEP (Figure 4B). Fluorescence lifetime measurement also supported the occurrence of FRET process in the BlueCy-TPB/PtOEP NPs (Table S3): Average lifetime of BlueCy-TPB decreased from $1.10 \pm 0.09$ ns to $0.87 \pm 0.07$ ns in the presence of PtOEP, in line with the intensity-based steady-state measurements. The 
presence of acceptor decreased decay times only for medium- and long-lived components (to 0.63 and $1.42 \mathrm{~ns}$, respectively), which corroborates with the FRET processes occurring on a similar time scale.
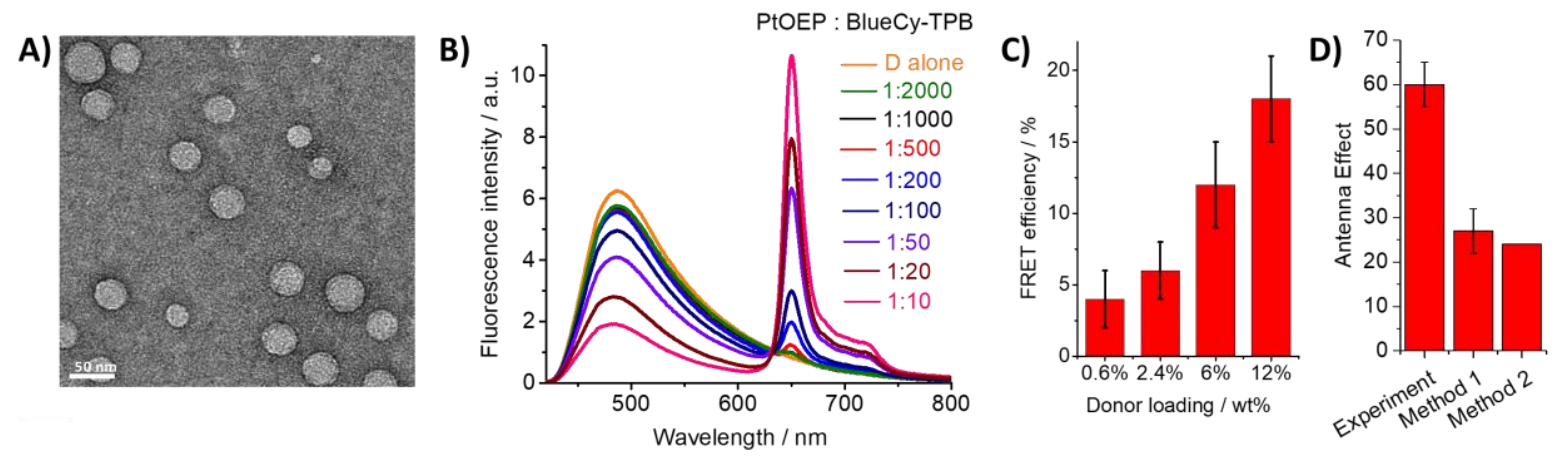

Figure 4. Characterization of FRET NPs. A) TEM image of BlueCy-TPB/PtOEP NP. B) Emission spectra of BlueCy-TPB/PtOEP NPs loaded with different amounts of the acceptor dye (PtOEP) and constant (12 wt\%, $100 \mathrm{mM}$ ) BlueCy-TPB donor loading. C) Plot of FRET efficiency of NPs with varied donor but constant acceptor (0.071 wt $\%, 1 \mathrm{mM})$ loading. (D) Antenna effect obtained for optimized FRET NPs (12 wt\% Donor, Donor : Acceptor ratio 100:1) obtained experimentally (from excitation spectra), based on calculation based on experimental FRET efficiency (Method 1) and based on calculation based on theoretical FRET efficiency (Method 2).

Remarkably, an increase in the donor loading from $0.6 \%$ to $12 \%$ for a constant acceptor (PtOEP) loading of $0.071 \mathrm{wt} \%(1 \mathrm{mM})$ also resulted in a growth of FRET efficiency from $4 \%$ to $18 \%$ (Figure 4C). These observations indicate the cooperative effect of the donor dyes, showing the light-harvesting phenomenon. According to our earlier studies, at higher dye loading, the donor dyes start to communicate, so that the excitation energy undergoes fast migration up to the energy acceptor. ${ }^{[24]}$ By analyzing excitation spectra of the FRET NPs (12 wt\% BlueCy-TPB) at the emission wavelength of the acceptor, we found that the antenna effect $(A E)$ was $60 \pm 5$. This indicates that with the light-harvesting process the acceptor emission can be amplified up to 60 -fold. The $A E$ value is directly linked to number of donors 
$\left(n_{D}\right)$ and acceptors $\left(n_{A}\right)$, their corresponding extinction coefficients ( $\varepsilon_{D}$ and $\varepsilon_{A}$, respectively) and FRET efficiency $(E): A E=n_{D \times} \varepsilon_{D} \times E /\left(n_{A} \times \varepsilon_{A} \times 100\right) .{ }^{[34]}$ Using FRET efficiency based on donor intensity changes, the estimated value of $\mathrm{AE}=2000 \times 67000 \times 18 /(20 \times 45000 \times 100)=27$ (method 1, Figure 4D). It is significantly lower than the experimental value, indicating that observed FRET efficiency is underestimated and should be $\sim 40 \%$ for $\mathrm{AE}=60$. The donor intensity does not take into account the dark states of self-quenched dyes that contribute to FRET without emitting in the donor channel. ${ }^{[35]}$ Then, we made a theoretical calculation of FRET efficiency, assuming a multi-chromophoric system with randomly distributed noncommunicating donors. ${ }^{[36]}$ Based on overlap integral between emission band of donor NPs and PtOEP absorption band, the calculated Forster radius was found to be $3.6 \mathrm{~nm}$. For 2000 donors and 20 acceptors confined in 40-nm particle, the theoretical FRET efficiency was 16\%, giving even lower estimated $\mathrm{AE}=24$ (method 2, Figure 4D). The much higher experimental AE value is probably linked to donor-donor excitation energy transfer, which ensures energy migration within multiple donors up to reaching the proximal acceptor. ${ }^{[24,25 b]}$

To examine the response of our FRET NPs, consisting A:D loading ratio of 1:100, to molecular oxygen, we exposed their suspensions in buffer to the flow of oxygen (oxygenated) and nitrogen (deoxygenated) gases. The observed emission at $650 \mathrm{~nm}$ for the FRET NPs in air-saturated solution got quenched in the oxygenated conditions, whereas it was significantly enhanced after the nitrogen purging (Figure 5A). Oxygenation/deoxygenation conditions produced strong effect on the acceptor emission, with almost negligible influence on the donor one. This result indicates that the observed PtOEP emission corresponds to phosphorescence, which is in line with the observed long acceptor emission lifetimes on the time scale of microseconds (Figure 5D) and the literature data for PtOEP. ${ }^{[37]}$ Observation of phosphoresce at room temperature is remarkable phenomenon that attracted strong attention recently. ${ }^{[38]}$ In our NPs, it was observed for two reasons. First, oxygen diffusion is largely slowed down in polymers (diffusion coefficient is $2.7-5.5 \times 10^{-9} \mathrm{~cm}^{2} \mathrm{~s}^{-1}$ in PMMA vs $2.5 \times 10^{-5}$ 
$\mathrm{cm}^{2} \mathrm{~s}^{-1}$ in water), ${ }^{[11 \mathrm{a}, 39]}$ which decreases collisional quenching of PtOEP triplet state, thus favoring its phosphorescence. Second reason is the light-harvesting phenomenon that amplifies emission of PtOEP. Importantly, oxygenation produced 90\% change in the ratio between donor fluorescence and acceptor phosphorescence, showing high sensitivity of the oxygen nanoprobes.

To further prove that the enhanced phosphorescence from PtOEP arise due to FRET from the BlueCy-TPB donor, we have carried out a control experiment with NPs containing 0.071 wt\% (1 mM) of PtOEP alone. In comparison to FRET NPs, PtOEP-loaded NPs showed negligible phosphorescence signal when excited at $405 \mathrm{~nm}$ or $535 \mathrm{~nm}$, corresponding to direct excitation of the acceptor (Figure S9). The latter confirms that the observed amplified phosphorescence signal in FRET NPs is due to the light-harvesting phenomenon, where multiple donors pump the excitation energy to few acceptors. Even in the deoxygenated condition, control PtOEP-loaded NPs showed 50-fold weaker phosphorescence when compared to the FRET NPs (Figure S9), in line with the observed high value of antenna effect. Finally, the reversibility of the system has been checked by the alternate purging of oxygen and nitrogen that showed stable and reversible ratiometric response of the FRET NPs (Figure 5B).
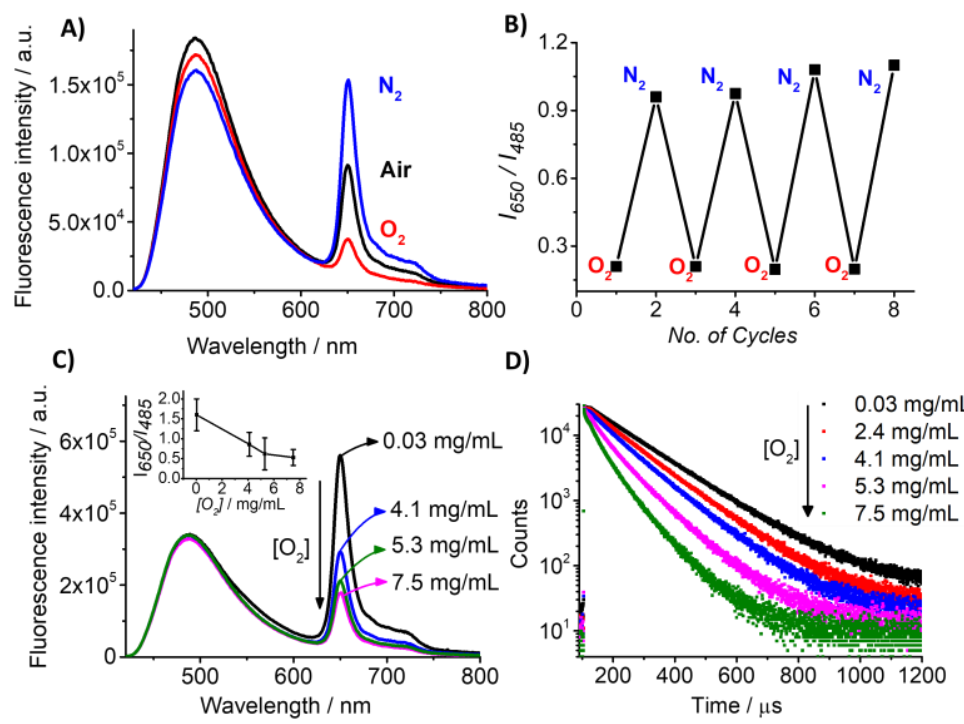
Figure 5. Oxygen sensing experiments using BlueCy-TPB/PtOEP NPs in phosphate buffer solution at $\mathrm{pH}$ 7.4. A) Ratiometric response in emission spectra of BlueCy-TPB/PtOEP NPs towards the change in oxygen concentration in the solution. Oxygen concentration was varied by purging oxygen and nitrogen gases. B) Ratiometric plot of emission intensities between acceptor and donor to the number of alternative purging of oxygen and nitrogen, to confirm the stability and reversibility of the BlueCy-TPB/PtOEP NPs as the oxygen probe. Changes in C) emission spectra and D) phosphorescence lifetime of BlueCy-TPB/PtOEP NPs by the successive variation of oxygen concentration in the solution.

The quantitative detection of molecular oxygen was then carried out by monitoring the ratiometric emission response of the BlueCy-TPB/PtOEP nanoprobe with regard to the successive decrease in oxygen concentration in the solution, using sodium sulfite $\left(\mathrm{Na}_{2} \mathrm{SO}_{3}\right)$, a well-known oxygen scavenger. ${ }^{[40]}$ By decreasing the oxygen concentration of the solution from $7.5 \mathrm{mg} / \mathrm{mL}$ to $0.03 \mathrm{mg} / \mathrm{mL}$, quantified by an optical probe for dissolved oxygen, we observed a gradual ratio change through enhancement in the acceptor phosphorescence, without altering the donor fluorescence (Figure 5C). This decrease in the oxygen concentration also produced a gradual increase in the phosphorescence lifetime of the acceptor from $60 \mu$ s to $138 \mu$ s (Figure 5D, Table S5). The ratiometric response of BlueCyTPB/PtOEP NPs to oxygen in the presence of BSA (48 $\mu \mathrm{M})$ and FBS (10\%), components of biological media, was nearly the same as in phosphate buffer (Figure S10). The observed invariant FRET and the ratiometric response to oxygen show that both donor and acceptor components are well encapsulated inside PMMA-MA NPs, without their detectable leakage, which makes this nanoprobe compatible with biological media.

Then, to evaluate ratiometric response of the nanoprobe to oxygen at the single-particle level, the immobilized BlueCy-TPB/PtOEP NPs (12 wt $\%$, PMMA-MA) at the glass surface were imaged with a microscope by collecting signals simultaneously at the donor (490-560 nm) and acceptor channels $(640-670 \mathrm{~nm}$ ) after exciting the donor (at $395 \mathrm{~nm}$ ). The control NPs (BlueCy-TPB) without acceptor appeared as very bright spots in the donor channel with poor 
emission in the acceptor channel. Interestingly, for the BlueCy-TPB/PtOEP NPs, we were able to detect the single-particle phosphorescence in the acceptor channel even under airsaturated conditions (Figure 6). The decrease in the intensity at the donor channel compared to the control sample (BlueCy-TPB alone), confirmed FRET from BlueCy-TPB to PtOEP. In the deoxygenated conditions (with sodium sulfite), the emission in the acceptor channel drastically increased and became higher than the donor channel, so that the pseudo-color in the merged images changed from green to yellow. The distinct changes in the two-color merge and ratiometric images (Figure 6, Figure S11) suggest the ability of our nanoprobe to operate as the oxygen sensor at the single-particle level. This become possible thanks to the light-harvesting process providing $\sim 60$-fold phosphorescence amplification of 20 encapsulated PtOEP dye in the NP, leading single-particle phosphoresce equivalent to $\sim 1200$ PtOEP dyes.

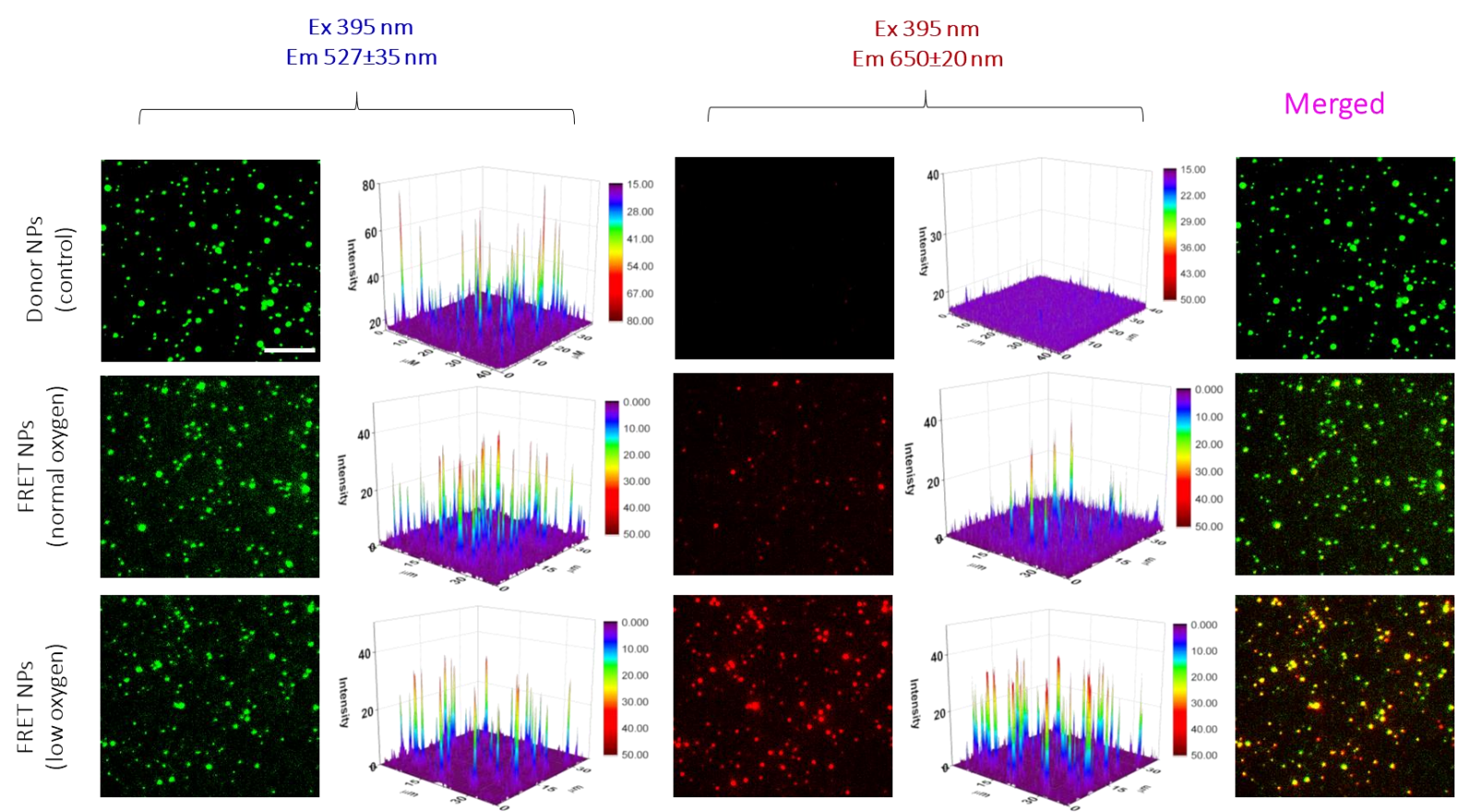

Figure 6. Single-particle evaluation of BlueCy-TPB/PtOEP NPs under wide-field fluorescence microscopy. The microscopic images and the corresponding 3D representation of the donor and acceptor channel for BlueCy-TPB (Donor NPs) and BlueCy-TPB/PtOEP NPs (FRET NPs) after the excitation at $395 \mathrm{~nm}$, in the air saturated and deoxygenated solutions. Scale bar; $10 \mu \mathrm{M}$. 


\subsection{Validation in live cells and phototoxicity}

After incubation with HeLa cells for $3 \mathrm{~h}$ at $37^{\circ} \mathrm{C}$, nanoprobe showed dotted emission in both donor and acceptor channels located inside the cells. This dotted emission is in agreement with the previous studies on polymeric and other NPs, ${ }^{[13 b-d, 17 b, 26]}$ suggesting localization of the nanoprobe in the endosomes and lysosomes. Indeed, it is well established that NPs below $100 \mathrm{~nm}$ enter cells by endocytosis. ${ }^{[41]}$ The presence of signal in the acceptor channel, colocalized with the donor channel (Figure 7A), as in our single-particle studies (Figure 6), suggested that the nanoprobe remained intact inside the cells, in line with the observed stability FRET nanoprobe in BSA and serum (Figure S10).

High brightness of the nanoprobe with amplified phosphorescence of PtOEP per particle allowed us to observe detectable signal in the acceptor channel at very low concentration of PtOEP (1 nM) in cells. This low concentration should significantly decrease phototoxicity of our system compared to PtOEP alone. To verify this, we incubated HeLa cells with our nanoprobe or the PtOEP dye and irradiated under same excitation power at their respective excitation wavelengths (395 $\mathrm{nm}$ for NPs and $550 \mathrm{~nm}$ for the PtOEP). Remarkably, to obtain the similar signal-to-background ratio at the phosphorescence (acceptor) channel, 500-fold higher concentration of PtOEP alone was needed (500 nM) compared to that used with the nanoprobe. This large concentration difference is in line $\sim 1200$-fold higher phosphorescence brightness of the nanoprobe compared to PtOEP dye. In these conditions, 5 min irradiation resulted in dramatic morphological alternations in cells incubated with PtOEP dye (Figure 7B), indicating its high cytotoxicity. In sharp contrast, no signs of cytotoxicity were detected in cells incubated with nanoprobe (Figure 7C). The latter result can be explained by 500 -fold lower effective concentration of PtOEP photosensitizer used with the nanoprobe in cells as well as the shielding effect of the polymer particle that physically separates the encapsulated photosensitizer from the cellular environment. 


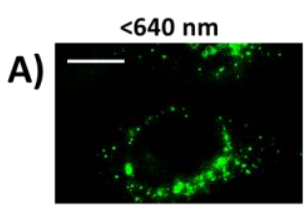

B)

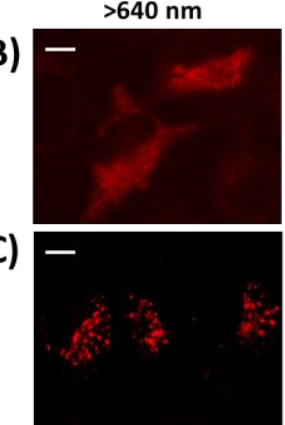

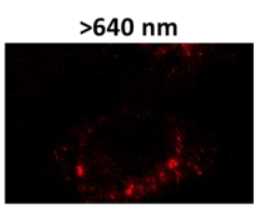

DIC

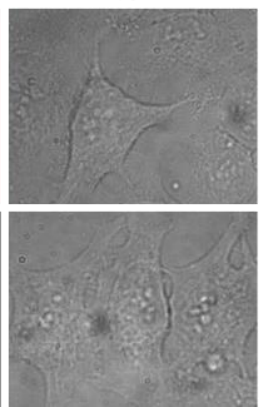

Composite

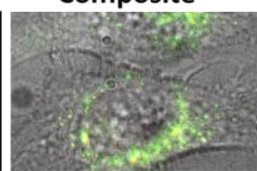

DIC, irradiated

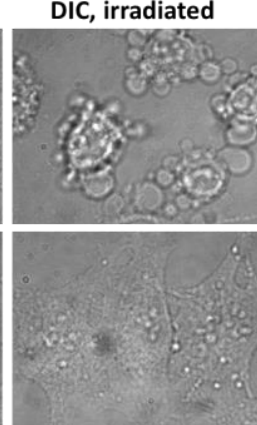

Figure 7. Internalization of nanoprobes and phototoxicity test. A) Fluorescence microscopy images of HeLa cells after incubation for $3 \mathrm{~h}$ with BlueCy-TPB/PtOEP nanoprobe (1 nM of PtOEP) at the donor $(<640 \mathrm{~nm})$ and acceptor $(>640 \mathrm{~nm})$ channels and their composite with bright-field images. B,C) Comparison of phototoxicity under the microscope for PtOEP dye alone (B) vs BlueCy-TPB/PtOEP NPs (C). B) Microscopic images (acceptor channel) and bright field images of HeLa cells after incubation for $3 \mathrm{~h}$ with PtOEP NPs (500 $\mathrm{nM}$ ) and irradiation at $550 \mathrm{~nm}$ for $5 \mathrm{~min}$. C) Corresponding images for BlueCy-TPB/PtOEP NPs (1 $\mathrm{nM}$ of PtOEP), after $5 \mathrm{~min}$ irradiation at $395 \mathrm{~nm}$. The irradiation power density was kept the same $\left(10 \mathrm{~W} \mathrm{~cm}^{-2}\right)$ for both excitation wavelengths; the concentration of PtOEP dye alone was increased in order to reach signal intensity values similar to that for the nanoprobe. Scale bars: $10 \mu \mathrm{M}$.

\subsection{Imaging oxygen gradients in cancer cells}

Then, in order to model hypoxia in cells, we designed a setup with a stable gradient of oxygen based on microfluidic plate ( $\mu$-ibidi) compatible with cell culture and fluorescence microscopy. From one outlet of the 50-mm long channel, the oxygen scavenger was added and the outlet was sealed, creating an oxygen restricted area, while the other end was exposed to air, providing oxygen abundant area (Figure 8A). Initially, we have optimized the addition of the oxygen scavenger into this microfluidic setup by using sodium dithionite reducing agent and Nile Red, presenting a chromogenic RedOx behavior (Figure S12). ${ }^{[42]}$ Then, using the optimized protocol we applied oxygen gradient in the microfluidic chamber with HeLa 
cells seeded and incubated for $3 \mathrm{~h}$ with our nanoprobes. Initially, using the two color detection mode, we imaged both ends of the chamber. Remarkably, a strong fluorescence signal from the FRET donor channel and a low signal from the acceptor channel were observed in the oxygen abundant area, whereas in the oxygen restricted end, enhanced phosphorescence at the acceptor channel was observed (Figure 8B), corresponding to significantly stronger acceptor/donor ratio in the hypoxic region (Figure $8 \mathrm{C}$ ). This result shows that our nanoprobe exhibits ratiometric response to dissolved oxygen directly inside the cells.
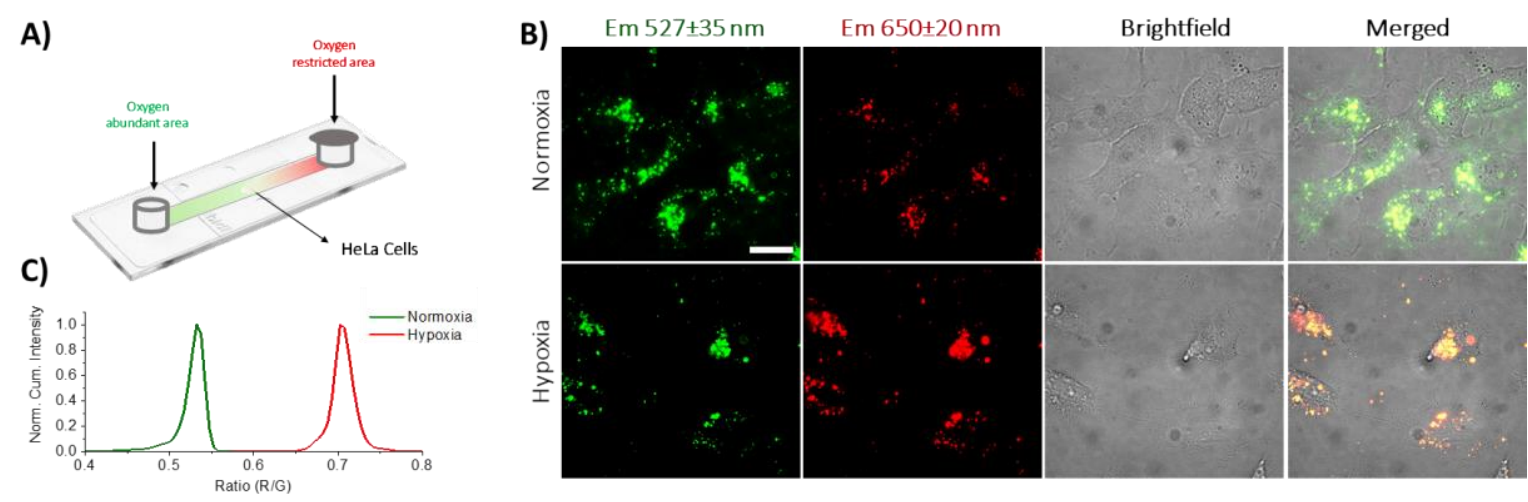

D)

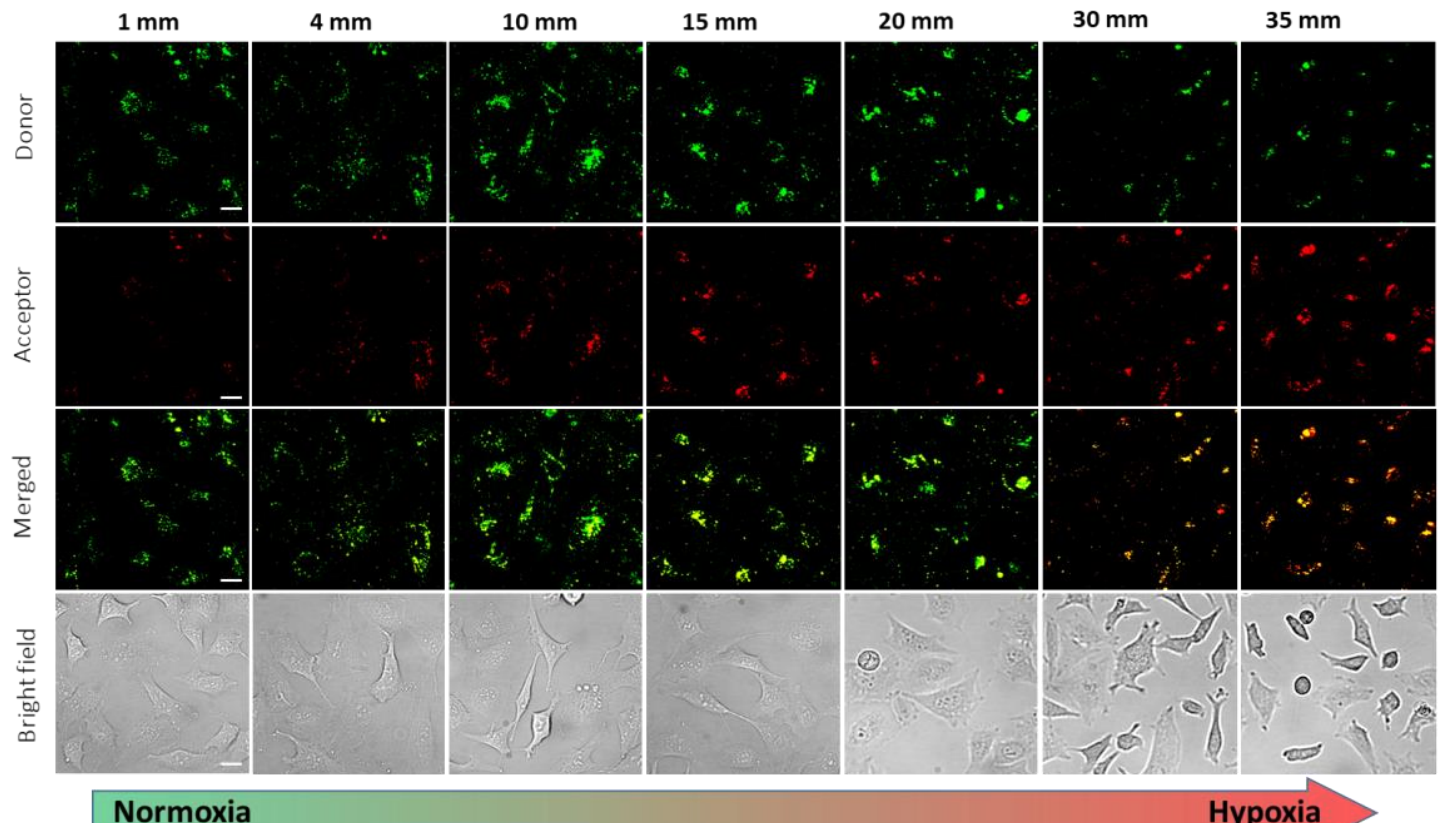

E)

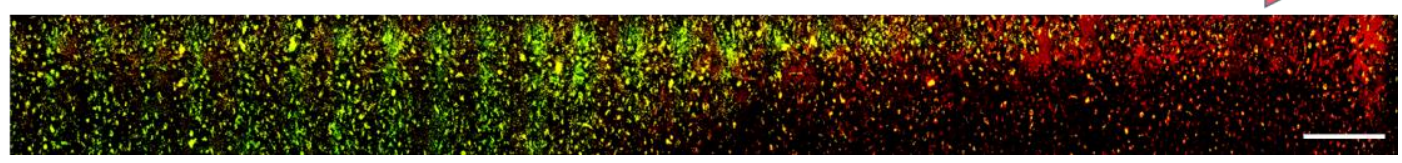

Figure 8. In vitro imaging of oxygen gradient in a microfluidic plate using BlueCyTPB/PtOEP NPs with wide-field fluorescence microscopy. A) Schematic representation of stable oxygen gradient generation in the microfluidic slide. B) Fluorescence microscopic 
images of HeLa cells after incubated for $3 \mathrm{~h}$ with BlueCy-TPB/PtOEP NPs. The images obtained in the different channels are shown (green, red, bright field and merged) in the oxygen abundant area (normoxia) and in oxygen restricted area (hypoxia) of the $\mu$-slide. Scale bar: $10 \mu \mathrm{m} . \mathrm{C})$ Acceptor-to-donor (Red/Green, R/G) ratio distribution plots derived from images of the corresponding normoxia and hypoxia regions. D) Top panel shows the step wise scan along the oxygen gradient in $\mu$-slide from normoxia and hypoxia region. Images are obtained after analyzing individual images shown at the mentioned distances from one end to other end. Scale bar: $20 \mu \mathrm{M}$. E) Large-scan image from one end to other end of the $\mu$-slide using 10x objective, which clearly depicts the oxygen gradient. Scale bar: $500 \mu \mathrm{M}$.

To further map the gradient of oxygen in HeLa cells within the microfluidic channel, we performed multiple images with $1 \mathrm{~mm}$ interval along the channel from the oxygen abundant to oxygen-restricted end. Within the defined length, we observed the gradual enhancement in the phosphorescence at the acceptor channel, accompanied by some decrease in the donor channel (Figure 8D). The gradient between normoxic and hypoxic regions was clearly visible in the merged images as a gradual change in emission color from green to yellow and then orange-red (Figure 8D). Finally, a large scale "panorama" two-color image of the microfluidic channel revealed a profile of this oxygen gradient (Figure $8 \mathrm{E}$ ).

\section{Conclusion}

Existing optical molecular probes for dissolved oxygen suffer from a number of fundamental limitations: (i) limited brightness of used phosphorescent dyes; (ii) need for complex detection schemes based on fluorescence lifetime and (iii) intrinsic photo-toxicity of phosphorescent dyes because of singlet-oxygen generation. Here, we introduce organic polymeric nanomaterials that undergo efficient FRET from large ensemble of encapsulated energy donor dyes (light-harvesting nanoantenna) to oxygen-sensitive phosphorescent unit and function as ratiometric nanoprobes for dissolved oxygen, which overcome the mentioned limitations. The light-harvesting nanoantenna particle is composed of a specially designed 
blue cyanine with bulky fluorinated counterions encapsulated inside poly(methyl methacrylate-co-methacrylic acid) (PMMA-MA) NPs. The resulted $40 \mathrm{~nm}$ dye-loaded NPs encapsulating ca. 2000 dyes with good fluorescence quantum yield (17\%) showed 70-fold higher brightness than QD525. Then, encapsulation into this light-harvesting nanoantenna of an oxygen-sensitive acceptor (PtOEP) at low concentration (1:100 acceptor : donor ratio) result in efficient FRET, so that the NPs emits both fluorescence of the donor and phosphorescence of the acceptor. The relative intensity of the phosphorescence band increases with decrease in oxygen concentration, which enables quantitative ratiometric measurements of oxygen in solution. Light-harvesting from bright fluorescent nanoparticle leads to $\sim 60$-fold amplification of PtOEP phosphorescence at room temperature and makes single particles as bright as 1200 PtOEP. Our approach with light-harvesting dye-loaded polymeric NPs is different from earlier studies on conjugated polymer NPs that also exploited FRET to PtOEP, ${ }^{[18 b]}$ because we aimed to achieve an efficient FRET with minimal amount of encapsulated acceptor. Indeed, in the previous representative work, ${ }^{[18 b]}$ bright phosphorescence of NPs was achieved by encapsulating 5500 PtOEP acceptors per particle, whereas in the present work, phosphorescence equivalent of $>1000$ dyes per particle was achieved with only 20 PtOEP acceptors. This amplified phosphorescence of the nanoprobe enables detection of dissolved oxygen at the single particle level and ratiometric imaging oxygen in cells at ultra-low concentration of encapsulated PtOEP $(1 \mathrm{nM})$. As a result, the obtained NPs do not show any sign of phototoxicity even after 5 min irradiation of the nanoprobes. By contrast, free PtOEP dye, which required much higher concentration (500 $\mathrm{nM}$ ) to achieve comparable phosphorescence signal from the cells showed strong phototoxicity. The developed nanoprobe was successfully applied to ratiometric imaging in a culture of cancer cells of stable oxygen gradient generated by a microfluidic setup. The high brightness, the ratiometric response and low phototoxicity make the developed nanomaterial a promising tool for imaging hypoxia, which is ubiquitously present in numerous pathologies. 


\section{Experimental Section}

The Experimental Section is available at the Supporting Information.

\section{Supporting Information}

Supporting Information is available from the Wiley Online Library or from the author.

\section{Acknowledgements}

This work was supported by Marie Curie post-doctoral research grant (MSCA-H2020-IF, NanoOxySens 705826) and European Research Council ERC Consolidator grant BrightSens 648528. P.A. acknowledges Senior Postdoctoral Fellowship grant sponsored under RUSA 2.0. The authors thank A. Russer and C. Crucifix for help with electron microscopy, K. Trofymchuk for help with theoretical FRET calculations, L. Richert for help with TCSPC measurements, A. Reisch for fruitful discussions and J. Valanciunaite for technical support.

\section{Conflict of interest}

The authors declare no conflict of interest.

Received: ((will be filled in by the editorial staff))

Revised: ((will be filled in by the editorial staff))

Published online: ((will be filled in by the editorial staff))

\section{References}

[1] a) T. Acker, H. Acker, Journal of Experimental Biology 2004, 207, 3171; b) José López-Barneo, a. Ricardo Pardal, P. Ortega-Sáenz, Annual Review of Physiology 2001, 63, 259.

[2] a) P. Carmeliet, et al., Nature 1998, 394, 485; b) D. Hanahan, R. A. Weinberg, Cell 2000, 100, 57; c) M. Höckel, K. Schlenger, S. Höckel, B. Aral, U. Schäffer, P. Vaupel, International Journal of Cancer 1998, 79, 365; d) M. Höckel, P. Vaupel, JNCI: 
Journal of the National Cancer Institute 2001, 93, 266; e) M.-C. Hung, G. B. Mills, D. Yu, Nature Medicine 2009, 15, 246; f) P. Vaupel, F. Kallinowski, P. Okunieff, Cancer Research 1989, 49, 6449.

[3] T. M. Freeman, W. R. Seitz, Analytical Chemistry 1981, 53, 98.

[4] D.-H. Zhao, S. Palanisamy, S.-M. Chen, Int. J. Electrochem. Sci. 2015, 10, 10038

[5] a) D. B. Papkovsky, R. I. Dmitriev, Chemical Society Reviews 2013, 42, 8700; b) X.-d. Wang, O. S. Wolfbeis, Chemical Society Reviews 2014, 43, 3666.

[6] a) C. McDonagh, B. D. MacCraith, A. K. McEvoy, Analytical Chemistry 1998, 70, 45;

b) C. Preininger, I. Klimant, O. S. Wolfbeis, Analytical Chemistry 1994, 66, 1841; c)

Z. Rosenzweig, R. Kopelman, Analytical Chemistry 1995, 67, 2650.

[7] a) M. Pawlowski, D. F. Wilson, in Oxygen Transport to Tissue XIII (Eds.: T. K. Goldstick, M. McCabe, D. J. Maguire), Springer US, Boston, MA, 1992, pp. 179; b) W. Rumsey, J. Vanderkooi, D. Wilson, Science 1988, 241, 1649; c) J. M. Vanderkooi, D. F. Wilson, in Oxygen Transport to Tissue VIII (Ed.: I. S. Longmuir), Springer US, Boston, MA, 1986, pp. 189; d) D. F. Wilson, in Oxygen Transport to Tissue XIV (Eds.: W. Erdmann, D. F. Bruley), Springer US, Boston, MA, 1992, pp. 195.

[8] a) J. N. Demas, B. A. DeGraff, Analytical Chemistry 1991, 63, 829A; b) I. Dunphy, S. A. Vinogradov, D. F. Wilson, Analytical Biochemistry 2002, 310, 191; c) H. Shi, X. Ma, Q. Zhao, B. Liu, Q. Qu, Z. An, Y. Zhao, W. Huang, Advanced Functional Materials 2014, 24, 4823.

[9] a) A. Fercher, S. M. Borisov, A. V. Zhdanov, I. Klimant, D. B. Papkovsky, ACS Nano 2011, 5, 5499; b) X.-H. Wang, H.-S. Peng, L. Yang, F.-T. You, F. Teng, L.-L. Hou, O. S. Wolfbeis, Angewandte Chemie International Edition 2014, 53, 12471.

[10] Y. Feng, J. Cheng, L. Zhou, X. Zhou, H. Xiang, Analyst 2012, 137, 4885.

[11] a) R. P. Briñas, T. Troxler, R. M. Hochstrasser, S. A. Vinogradov, Journal of the American Chemical Society 2005, 127, 11851; b) E. Roussakis, J. A. Spencer, C. P. Lin, S. A. Vinogradov, Analytical Chemistry 2014, 86, 5937.

[12] a) L. Feng, C. Zhu, H. Yuan, L. Liu, F. Lv, S. Wang, Chemical Society Reviews 2013, 42, 6620; b) Y. Jiang, J. McNeill, Chemical Reviews 2017, 117, 838; c) J. Pecher, S. Mecking, Chemical Reviews 2010, 110, 6260; d) K.-Y. Pu, B. Liu, Advanced Functional Materials 2011, 21, 3408; e) C. Wu, D. T. Chiu, Angewandte Chemie International Edition 2013, 52, 3086.

[13] a) N. Hildebrandt, C. M. Spillmann, W. R. Algar, T. Pons, M. H. Stewart, E. Oh, K. Susumu, S. A. Díaz, J. B. Delehanty, I. L. Medintz, Chemical Reviews 2017, 117, 536; b) A. M. Keller, Y. Ghosh, M. S. DeVore, M. E. Phipps, M. H. Stewart, B. S. Wilson, D. S. Lidke, J. A. Hollingsworth, J. H. Werner, Advanced Functional Materials 2014, 24, 4796; c) A. Shamirian, H. Samareh Afsari, A. Hassan, L. W. Miller, P. T. Snee, ACS Sensors 2016, 1, 1244; d) K. D. Wegner, N. Hildebrandt, Chemical Society Reviews 2015, 44, 4792.

[14] a) S. Y. Lim, W. Shen, Z. Gao, Chemical Society Reviews 2015, 44, 362; b) X. T. Zheng, A. Ananthanarayanan, K. Q. Luo, P. Chen, Small 2015, 11, 1620.

[15] a) S. W. Bae, W. Tan, J.-I. Hong, Chemical Communications 2012, 48, 2270; b) D. Genovese, E. Rampazzo, S. Bonacchi, M. Montalti, N. Zaccheroni, L. Prodi, Nanoscale 2014, 6, 3022; c) M. Montalti, L. Prodi, E. Rampazzo, N. Zaccheroni, Chemical Society Reviews 2014, 43, 4243.

[16] a) J. Mei, N. L. C. Leung, R. T. K. Kwok, J. W. Y. Lam, B. Z. Tang, Chemical Reviews 2015, 115, 11718; b) K. Li, B. Liu, Chemical Society Reviews 2014, 43, 6570; c) J. Geng, K. Li, W. Qin, L. Ma, G. G. Gurzadyan, B. Z. Tang, B. Liu, Small 2013, 9, 2012; d) W.-C. Wu, et al., Advanced Functional Materials 2010, 20, 1413; e) X. Yan, et al., ACS Applied Materials \& Interfaces 2018, 10, 25154; f) Y. Yang, F. An, 
Z. Liu, X. Zhang, M. Zhou, W. Li, X. Hao, C.-s. Lee, X. Zhang, Biomaterials 2012, 33, 7803 .

[17] a) A. Reisch, A. S. Klymchenko, Small 2016, 12, 1968; b) A. Reisch, P. Didier, L. Richert, S. Oncul, Y. Arntz, Y. Mély, A. S. Klymchenko, Nature Communications 2014, 5, 4089; c) A. Reisch, A. Runser, Y. Arntz, Y. Mély, A. S. Klymchenko, ACS Nano 2015, 9, 5104; d) A. Wagh, F. Jyoti, S. Mallik, S. Qian, E. Leclerc, B. Law, Small 2013, 9, 2129; e) K. Trofymchuk, A. Reisch, I. Shulov, Y. Mély, A. S. Klymchenko, Nanoscale 2014, 6, 12934.

[18] a) R. I. Dmitriev, S. M. Borisov, H. Düssmann, S. Sun, B. J. Müller, J. Prehn, V. P. Baklaushev, I. Klimant, D. B. Papkovsky, ACS Nano 2015, 9, 5275; b) C. Wu, B. Bull, K. Christensen, J. McNeill, Angewandte Chemie International Edition 2009, 48, 2741; c) Q. Zhao, X. Zhou, T. Cao, K. Y. Zhang, L. Yang, S. Liu, H. Liang, H. Yang, F. Li, W. Huang, Chemical Science 2015, 6, 1825; d) X. Zhou, et al., Advanced Science 2016, 3, 1500155.

[19] X.-d. Wang, H. H. Gorris, J. A. Stolwijk, R. J. Meier, D. B. M. Groegel, J. Wegener, O. S. Wolfbeis, Chemical Science 2011, 2, 901.

[20] A. Byrne, J. Jacobs, C. S. Burke, A. Martin, A. Heise, T. E. Keyes, Analyst 2017, 142, 3400 .

[21] Y.-Y. Huang, Y. Tian, X.-Q. Liu, Z. Niu, Q.-Z. Yang, V. Ramamurthy, C.-H. Tung, Y.-Z. Chen, L.-Z. Wu, Materials Chemistry Frontiers 2018, 2, 1893.

[22] a) J. Arnbjerg, A. Jiménez-Banzo, M. J. Paterson, S. Nonell, J. I. Borrell, O. Christiansen, P. R. Ogilby, Journal of the American Chemical Society 2007, 129, 5188; b) R. Bonnett, R. D. White, U. J. Winfield, M. C. Berenbaum, Biochem J 1989, 261, 277; c) S. C. Karunakaran, et al., ACS Chemical Biology 2013, 8, 127; d) H. Liu, H. Yang, X. Hao, H. Xu, Y. Lv, D. Xiao, H. Wang, Z. Tian, Small 2013, 9, 2639; e) J. F. Lovell, T. W. B. Liu, J. Chen, G. Zheng, Chemical Reviews 2010, 110, 2839; f) I. J. Macdonald, T. J. Dougherty, Journal of Porphyrins and Phthalocyanines 2001, 5, 105.

[23] A. Reisch, D. Heimburger, P. Ernst, A. Runser, P. Didier, D. Dujardin, A. S. Klymchenko, Advanced Functional Materials 2018, 28, 1805157.

[24] K. Trofymchuk, A. Reisch, P. Didier, F. Fras, P. Gilliot, Y. Mely, A. S. Klymchenko, Nature Photonics 2017, 11, 657.

[25] a) N. Melnychuk, A. S. Klymchenko, Journal of the American Chemical Society 2018, 140, 10856; b) N. Melnychuk, S. Egloff, A. Runser, A. Reisch, A. S. Klymchenko, Angewandte Chemie International Edition 2020, 59, 6811.

[26] B. Andreiuk, A. Reisch, M. Lindecker, G. Follain, N. Peyriéras, J. G. Goetz, A. S. Klymchenko, Small 2017, 13, 1701582.

[27] a) B. Andreiuk, A. Reisch, E. Bernhardt, A. S. Klymchenko, Chemistry - An Asian Journal 2019, 14, 836; b) B. Andreiuk, A. Reisch, V. G. Pivovarenko, A. S. Klymchenko, Materials Chemistry Frontiers 2017, 1, 2309; c) N. Adarsh, A. S. Klymchenko, Nanoscale 2019, 11, 13977.

[28] A. Reisch, K. Trofymchuk, A. Runser, G. Fleith, M. Rawiso, A. S. Klymchenko, ACS Applied Materials \& Interfaces 2017, 9, 43030.

[29] a) C. M. Lemon, P. N. Curtin, R. C. Somers, A. B. Greytak, R. M. Lanning, R. K. Jain, M. G. Bawendi, D. G. Nocera, Inorganic Chemistry 2014, 53, 1900; b) C. M. Lemon, E. Karnas, M. G. Bawendi, D. G. Nocera, Inorganic Chemistry 2013, 52, 10394; c) J. Liu, Y. Liu, W. Bu, J. Bu, Y. Sun, J. Du, J. Shi, Journal of the American Chemical Society 2014, 136, 9701; d) R. Xu, Y. Wang, X. Duan, K. Lu, D. Micheroni, A. Hu, W. Lin, Journal of the American Chemical Society 2016, 138, 2158.

[30] a) L. G. S. Brooker, G. H. Keyes, W. W. Williams, Journal of the American Chemical Society 1942, 64, 199; b) H. Yao, K. Ashiba, RSC Advances 2011, 1, 834; c) S. M. 
Yarmoluk, S. S. Lukashov, T. Y. Ogul'Chansky, M. Y. Losytskyy, O. S. Kornyushyna, Biopolymers 2001, 62, 219.

[31] a) X. Gu, et al., Advanced Materials 2018, 30, 1801065; b) Y. Hong, J. W. Y. Lam, B. Z. Tang, Chemical Society Reviews 2011, 40, 5361; c) J. Luo, et al., Chemical Communications 2001, 1740.

[32] a) T. L. Netzel, K. Nafisi, M. Zhao, J. R. Lenhard, I. Johnson, The Journal of Physical Chemistry 1995, 99, 17936; b) J. Nygren, N. Svanvik, M. Kubista, Biopolymers 1998, 46, 39.

[33] I. O. Aparin, N. Melnychuk, A. S. Klymchenko, Adv. Optical Mater. 2020, DOI: 10.1002/adom.202000027.

[34] J. G. Woller, J. K. Hannestad, B. Albinsson, Journal of the American Chemical Society 2013, 135, 2759.

[35] D. Genovese, S. Bonacchi, R. Juris, M. Montalti, L. Prodi, E. Rampazzo, N. Zaccheroni, Angewandte Chemie International Edition 2013, 52, 5965.

[36] in Principles of Fluorescence Spectroscopy (Ed.: J. R. Lakowicz), Springer US, Boston, MA, 2006, pp. 507.

[37] S. Izakura, W. Gu, R. Nishikubo, A. Saeki, The Journal of Physical Chemistry C 2018, $122,14425$.

[38] a) N. Gan, H. Shi, Z. An, W. Huang, Advanced Functional Materials 2018, 28, 1802657; b) Kenry, C. Chen, B. Liu, Nature Communications 2019, 10, 2111; c) Q. Li, Y. Tang, W. Hu, Z. Li, Small 2018, 14, 1801560; d) X.-F. Wang, H. Xiao, P.-Z. Chen, Q.-Z. Yang, B. Chen, C.-H. Tung, Y.-Z. Chen, L.-Z. Wu, Journal of the American Chemical Society 2019, 141, 5045; e) H. Wu, W. Chi, Z. Chen, G. Liu, L. Gu, A. K. Bindra, G. Yang, X. Liu, Y. Zhao, Advanced Functional Materials 2019, 29, 1970063.

[39] G. Shaw, Transactions of the Faraday Society 1967, 63, 2181.

[40] Z. Tao, J. Goodisman, A.-K. Souid, The Journal of Physical Chemistry A 2008, 112, 1511.

[41] S. Patel, J. Kim, M. Herrera, A. Mukherjee, A. V. Kabanov, G. Sahay, Advanced Drug Delivery Reviews 2019, 144, 90.

[42] O. A. Kucherak, S. Oncul, Z. Darwich, D. A. Yushchenko, Y. Arntz, P. Didier, Y. Mély, A. S. Klymchenko, Journal of the American Chemical Society 2010, 132, 4907. 


\section{The table of contents}

\section{Nanoparticle biosensor}

P. Ashokkumar, N. Adarsh, A. S. Klymchenko*

Ratiometric nanoparticle probe based on FRET-amplified phosphorescence for oxygen sensing with minimal phototoxicity

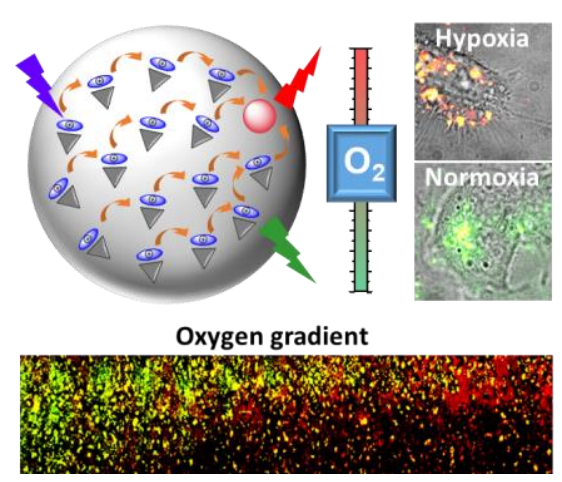

Luminescent oxygen-sensing nanoparticles are developed based on light-harvesting principle that ensures efficient FRET from thousands of donor fluorescent dyes to few oxygen-sensitive phosphorescent acceptors. Owing to high brightness, ratiometric response to dissolved oxygen and low photo-toxicity, the developed nanoprobe is applied to imaging of microfluidics-generated oxygen gradients in cancer cells. 